\title{
Medical education in the time of COVID-19
}

\author{
Diane B. Wayne', Marianne Green ${ }^{2}$, and Eric G. Neilson ${ }^{3^{*}}$ \\ ${ }^{1}$ Vice Dean for Education, Northwestern University Feinberg School of Medicine; ${ }^{2}$ Senior Associate Dean for Medical Education, Northwestern University Feinberg School of \\ Medicine; ${ }^{3}$ Vice President for Medical Affairs, Lewis and Landsberg Dean, Northwestern University Feinberg School of Medicine, Arthur J. Rubloff Building, Suite $12-109$, 420 \\ East Superior Street, Chicago, IL 60611
}

${ }^{*}$ Corresponding author. Email: egneilson@northwestern.edu

In current circumstances one rightfully wonders if persistence of SARS-Cov-2 will fundamentally alter the landscape of medical education and hospital training. Absent a vaccine, the prevalence of this virus adds to annual respiratory illnesses caused by seasonal influenza, respiratory syncytial virus, rhinoviruses, and other coronaviruses. Faced with a looming new-normal, many educators are ruminating on how best to ensure rigorous medical training that produces a steady stream of competent physicians.

By way of background, the umbrella of medical education covers a highly structured curriculum in a variety of pre-clinical and clinical environments whose architecture and requirements are set by the Liaison Committee for Medical Education (LCME) (1) and the Accreditation Council for Graduate Medical Education (ACGME) (2). These requirements reflect established habits for producing quality outcomes. Capricious changes to these requirements can alter the carat of each uncut gem matriculating to medical school. And for this reason, students follow inviolate course work to their doctorate. Graduates can apply for state licensure to become physicians after receiving accredited training as interns and residents. Only later when seen serving a public good are physicians fully vested professionals. Such training can last 7-10 years.

Modern training encompasses a well-thought-out system of educational milestones that are highly interactive, increasingly team-based, and guided by educators who have mastered their craft. Competent physicians are not born, they are made-taught to integrate the language of science with recent concepts of disease, diagnosis, treatment, and empathy. A gradual move to some outcome measures now provides a few entrustable professional activities and procedures students must master before residency training (3). Technology also plays an increasingly important role in teaching core clinical skills as simulation centers and computerized anatomy laboratories become more prevalent (4). Patient histories and physical examinations, how hospitals and medical records work, and the art of detective conversations can only be honed, however, in supervised settings that engage real patients.

This spring, medical education in many institutions experienced abrupt disruptions in the face of the COVID-19 pandemic. Teaching hospitals encountered a rapidly expanding illness, shortages of personal protective equipment (PPE), and growing concern for exposures to asymptomatic carriers. And at Northwestern Medicine our least experienced clinicians, the medical students, were removed temporarily from direct patient care. The pre-clinical curriculum was migrated online with evidence of similar or improved learning compared to prior years. Students on hospital rotations completed virtual clerkships and clinical skill assessments and participated in newly created on-line electives. Interdisciplinary faculty similarly launched COVID-19 courses focusing on the pathophysiology, diagnosis, and treatment of the infection, as well as the health disparities and ethical considerations associated with pandemics.

While marching along this curriculum, medical students also channeled their energy and concern through community service. They led donation efforts for PPE, set up food drives, participated in patient education at community sites, worked with clinicians to contact patients diagnosed with COVID-19 to assess their health status, and made food and prescription deliveries to senior citizens and others needing help across Chicago. Through these experiences, students learned about health inequities and social determinants of disease in a manner not easily addressed in the classroom.

Several months into this disruption our focus has shifted toward creating a new normal. While a return to contact patient care and teaching remains highly desirable, we are carefully evaluating various formats for delivering other parts of the curriculum. What seems certain is a return to a typical pre-COVID-19 teaching platform is unlikely and many creative changes are here to stay. Large-scale adoption of online education during the pandemic shows it is possible to achieve a number of teaching objectives virtually. Faculty previously resistant to technology-enhanced learning now have evidence of its ability to meet the needs of pre-clinical students who value adaptive and self-directed study; some may need to review specific content several times whereas others will proceed more rapidly. Augmented intelligence and machine learning will support this model by achieving the goal of a more tailored outcomes-based education. Undoubtedly, this 
undertaking will result in additional innovations, flexibility, and experimentation in areas such as anatomy, problembased learning. clinical skills education, assessment of student well-being, and mentorship or career advising.

Many improvements to medical education are a natural consequence of disruptive moments. As we reflect on the COVID-19 pandemic, changes to the medical curriculum that ensure more focus on infection control, pandemic modeling, population and public health, telemedicine, and health equity are desirable. New learners need modern tools to prepare for a response to unexpected medical events in the future. Additionally, we have witnessed the spreading value of resilience, grit, and tolerance for uncertainty on the front lines of patient care. We must continue to select for these qualities in future matriculants.

Despite the temptation for unfettered innovation, we also know some elements of the curriculum cannot change. The heroic actions of health care workers currently treating COVID-19 patients reaffirms professionalism and community service as core attributes of a well-taught student. Clinical competency also depends on reliable assessment tools that ensure our graduates are prepared to enter residency training with the knowledge and skills to provide safe and effective patient care.

Simon Sinek reminds us that "working hard for something we don't care about is called stress, while working hard for something we love is called passion." (5) The latter is achieved by witnessing selflessness and a desire to make a difference, core traits that inspired our students to pursue careers in medicine in the first place. As we look toward the future, medical education may never be the same again and our accrediting agencies will have to join in the adaptation. We now have an opportunity to create a better medical school experience with improved flexibility and outcomes that still ensures competence from this increasingly complex effort.

\section{REFERENCES AND NOTES}

1. LCME, Liaison Committee for Medical Education. https://lcme.org/

2. ACGME, Accreditation Council for Graduate Medical Education. https://www.acgme.org/

3. K. Lomis, J. M. Amiel, M. S. Ryan, K. Esposito, M. Green, A. Stagnaro-Green, J. Bull, G. C. Mejicano; AAMC Core EPAs for Entering Residency Pilot Team, Implementing an entrustable professional activities framework in undergraduate medical education: Early lessons from the AAMC core entrustable professional activities for entering residency pilot. Acad. Med. 92, 765-770 (2017). doi:10.1097/ACM.0000000000001543 Medline

4. T. Uchida, Y. S. Park, R. K. Ovitsh, J. Hojsak, D. Gowda, J. M. Farnan, M. Boyle, A. D. Blood, F. I. Achike, R. C. Silvestri, Approaches to teaching the physical exam to preclerkship medical students: Results of a national survey. Acad. Med. 94, 129134 (2019). doi:10.1097/ACM.0000000000002433 Medline

5. Simon Sinek, https://www.goalcast.com/2017/08/29/top-simon-sinek-quoteshard-truths-success/

Published First Release 05 June 2020

10.1126/sciadv.abc7110 